\title{
Article \\ B Cells with a Senescent-Associated Secretory Phenotype Accumulate in the Adipose Tissue of Individuals with Obesity
}

\author{
Daniela Frasca $^{1,2, *}$, Maria Romero ${ }^{1}$, Alain Diaz ${ }^{1}$, Denisse Garcia ${ }^{1}$, Seth Thaller ${ }^{3}$ and Bonnie B. Blomberg ${ }^{1,2}$ \\ 1 Department of Microbiology and Immunology, University of Miami Miller School of Medicine, \\ Miami, FL 33136, USA; mromero5@med.miami.edu (M.R.); a.diaz7@med.miami.edu (A.D.); \\ d.garcia24@umiami.edu (D.G.); bblomber@med.miami.edu (B.B.B.) \\ 2 Sylvester Comprehensive Cancer Center, Miami, FL 33136, USA \\ 3 Department of Surgery, Division of Plastic and Reconstructive Surgery, \\ University of Miami Miller School of Medicine, Miami, FL 33136, USA; sthaller@med.miami.edu \\ * Correspondence: dfrasca@med.miami.edu
}

Citation: Frasca, D.; Romero, M.;

Diaz, A.; Garcia, D.; Thaller, S.; Blomberg, B.B. B Cells with a Senescent-Associated Secretory Phenotype Accumulate in the Adipose Tissue of Individuals with Obesity. Int. J. Mol. Sci. 2021, 22, 1839 https://doi.org/10.3390/ijms22041839

Received: 15 January 2021

Accepted: 8 February 2021

Published: 12 February 2021

Publisher's Note: MDPI stays neutral with regard to jurisdictional claims in published maps and institutional affiliations.

Copyright: (c) 2021 by the authors. Licensee MDPI, Basel, Switzerland. This article is an open access article distributed under the terms and conditions of the Creative Commons Attribution (CC BY) license (https:// creativecommons.org/licenses/by/ $4.0 /)$.

\begin{abstract}
Senescent cells accumulate in the adipose tissue (AT) of individuals with obesity and secrete multiple factors that constitute the senescence-associated secretory phenotype (SASP). This paper aimed at the identification of B cells with a SASP phenotype in the AT, as compared to the peripheral blood, of individuals with obesity. Our results show increased expression of SASP markers in AT versus blood B cells, a phenotype associated with a hyper-metabolic profile necessary to support the increased immune activation of AT-derived B cells as compared to blood-derived B cells. This hyper-metabolic profile is needed for the secretion of the pro-inflammatory mediators (cytokines, chemokines, micro-RNAs) that fuel local and systemic inflammation.
\end{abstract}

Keywords: B cells; senescence; inflammation; obesity

\section{Introduction}

Cellular senescence indicates the irreversible arrest of cell proliferation that is induced by different stress-derived signals. It is mediated by the inhibition of cell cycle progression through $\mathrm{p} 16^{\mathrm{INK} 4}$ and/or the activation of cell cycle arrest through $\mathrm{p} 53 / \mathrm{p} 21$. Stressors inducing cell senescence include DNA damage, telomere shortening, radiation, reactive metabolites, mitogenic and metabolic stressors. Although senescent cells show changes in chromatin organization and gene expression, they remain metabolically active [1,2]. The senescence-associated secretory phenotype (SASP) of senescent cells is characterized by the secretion of soluble pro-inflammatory factors (cytokines, chemokines, micro-RNAs), soluble cytokine receptors (TNF receptors), non-protein soluble factors (nitric oxide), growth factors (EGF, VGEF, NGF) and extracellular matrix macromolecules (fibronectin, collagens, laminin) $[3,4]$. Senescent cells accumulate in the body during aging, promote tissue degeneration and malignant transformation, and lead to the development of inflammatory-based age-associated diseases $[5,6]$. Senescent cells also accumulate in the adipose tissue (AT) of mice and humans, and secrete multiple SASP factors that have been shown to induce cell death, increased local and systemic inflammation and recruitment of immune cells, leading to AT dysfunction, insulin resistance and type-2 diabetes [7-9].

Obesity is a condition associated with chronic low-grade systemic inflammation, known as inflammaging [10]. Inflammaging induces chronic immune activation (IA), functional impairment of immune cells and decreased immunity. Obesity and associated inflammation lead to several debilitating chronic diseases such as type-2 diabetes [11-13], cardiovascular disease [14], cancer [15], atherosclerosis [16], inflammatory bowel disease [17]. Obesity also leads to dysfunctional immunity and decreases the proportion of individuals responding to vaccination [18-20], as well as of patients achieving remission in response to therapy [21-23], which may further affect the outcome of infection, with obvious health consequences for the individual. It is not currently known if the obesity-driven 
impairment of immune function contributes to reduced clearance of senescent cells leading to their increased accumulation in the AT.

We have previously characterized the obesity-associated decrease in B cell function [18]. We have shown that unstimulated B cells from the peripheral blood of individuals with obesity, as compared to those from lean individuals, express higher levels of RNA and protein for multiple inflammaging-associated pro-inflammatory cytokines (e.g., TNF- $\alpha$ ). B cell intrinsic TNF- $\alpha$ positively correlates with serum TNF- $\alpha$ and both negatively correlate with $B$ cell function, measured by activation-induced cytidine deaminase (AID) after in vivo or in vitro stimulation with mitogens, antigens and vaccines. AID is the enzyme that regulates Ig (immunoglobulin) class switch recombination and somatic hypermutation [24], two processes leading to the generation of high affinity protective antibodies [25-27].

The accumulation of senescent $\mathrm{T}$ cells in the human AT under obesity conditions has been reported [28], but virtually nothing is known about the accumulation of senescent $B$ cells. In this paper, we have investigated senescent B cells present in the subcutaneous AT of patients undergoing weight reduction surgeries, as compared to those from the blood of individuals with obesity (age- and gender-matched), and we have characterized their SASP phenotype and their metabolic profile. Results show increased expression of SASP markers in AT versus blood B cells, a phenotype associated with a hyper-metabolic profile necessary to support increased IA of B cells from the AT as compared to those from the peripheral blood, including the production of several pro-inflammatory mediators that fuel local and systemic inflammation.

\section{Results}

2.1. Increased Expression of SASP Markers in B Cells from the AT of Individuals with Obesity as Compared to the Peripheral Blood

B cells were isolated from the peripheral blood of individuals with obesity using magnetic beads and from the AT of obese surgery patients using flow cytometry and cell sorting (as indicated in Materials and Methods, Section 4.4). After isolation, B cells were left unstimulated and were resuspended in TRIzol. The RNA was then extracted and the expression of SASP markers evaluated by qPCR. We measured RNA expression of the following pro-inflammatory markers: cytokines (TNF, IL-6), chemokines (IL-8), micro-RNAs, miRs (miR-155, miR-16, miR-181a) and cell cycle inhibitors and markers of proliferation arrest (p16 $6^{\mathrm{IN} 4}, \mathrm{p} 21^{\mathrm{CIP} 1 / \mathrm{WAF} 1}$ and $\left.\mathrm{p} 53\right)$. Figure 1 shows significantly higher expression of RNA for pro-inflammatory cytokines and chemokines (1A), for pro-inflammatory miRs (1B) and for p16 ${ }^{\mathrm{INK} 4}, \mathrm{p} 21^{\mathrm{CIP} 1 / \mathrm{WAF} 1}$ and p53 (1C) in B cells from the AT as compared to B cells from the peripheral blood.

Although the purpose of our study was to compare RNA levels of multiple SASP markers in B cells from the AT of surgery patients and from the blood of individuals with obesity, we also evaluated RNA expression of these SASP markers in B cells from the blood of lean individuals as compared to our cohort of obese individuals (age- and gendermatched). We found significantly increased RNA expression of all SASP markers in B cells from obese versus lean individuals: TNF, $0.38 \pm 0.03$ versus $0.17 \pm 0.03(p=0.0006)$; IL-6, $0.23 \pm 0.01$ versus $0.16 \pm 0.01(p=0.003) ; \mathrm{IL}-8,0.17 \pm 0.01$ versus $0.11 \pm 0.01(p=0.0008)$; miR-155, $0.23 \pm 0.03$ versus $0.09 \pm 0.02(p=0.001)$; miR-16, $0.31 \pm 0.03$ versus $0.11 \pm 0.02$ $(p=0.0009) ;$ miR-181a, $0.18 \pm 0.01$ versus $0.08 \pm 0.01(p=0.0009) ; \mathrm{p} 16^{\mathrm{INK} 4}, 0.28 \pm 0.02$ versus $0.15 \pm 0.02(p=0.001) ; \mathrm{p} 21^{\mathrm{CIP} 1 / \mathrm{WAF} 1}, 0.30 \pm 0.01$ versus $0.19 \pm 0.02(p=0.0001) ; \mathrm{p} 53$, $0.41 \pm 0.04$ versus $0.15 \pm 0.01(p=0.0002)$. These results altogether clearly indicate that the blood of individuals with obesity, as compared to the blood of lean individuals, is enriched in B cells expressing RNA for multiple SASP markers. These markers are expressed at even higher levels in B cells from the AT of surgery patients. 

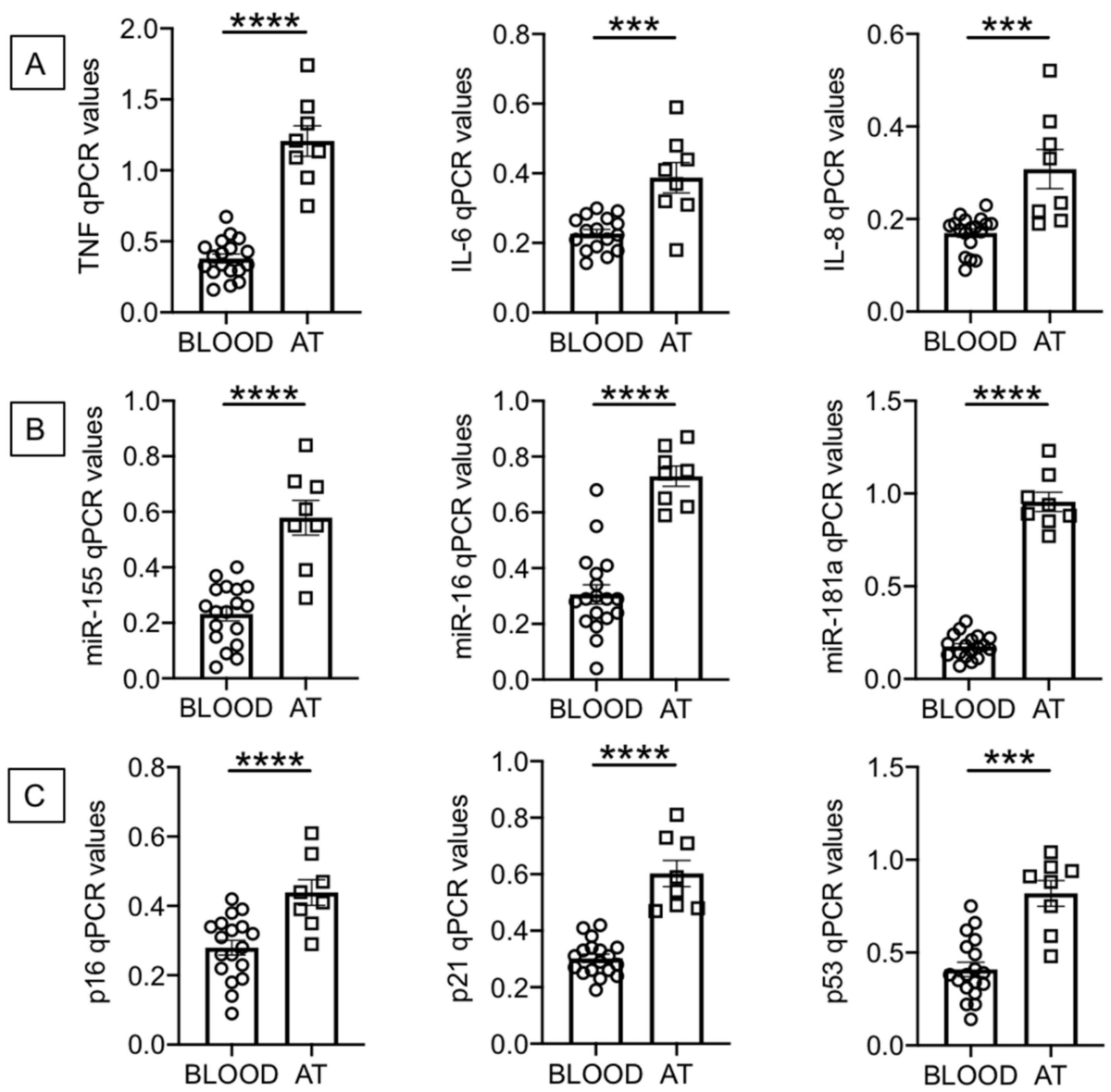

Figure 1. Increased expression of senescence-associated secretory phenotype (SASP) markers in B cells from the adipose tissue (AT), as compared to those from the peripheral blood, of individuals with obesity. B cells isolated from peripheral blood mononuclear cells (PBMC) and stromal vascular fraction (SVF) were resuspended in TRIzol, then the RNA was extracted and the expression of SASP markers detected by qPCR to evaluate expression of RNA for pro-inflammatory cytokines and chemokines (A), pro-inflammatory miRs (B), and cell cycle regulators p16 ${ }^{\mathrm{INK} 4}, \mathrm{p} 21^{\mathrm{CIP} 1 / \mathrm{WAF} 1}$ and p53 (C). qPCR values are measures of RNA expression of target genes, relative to the housekeeping genes GAPDH or U6 (for miRs quantification), calculated as $2^{-\Delta C t s}$. Mean comparisons between groups were performed by unpaired Student's $t$ test (two-tailed). ${ }^{* * *} p<0.001,{ }^{* * * *} p<0.0001$.

2.2. Increased Frequencies of Memory B Cells, and Decreased Frequencies of Nä̈ve B Cells, in the AT of Individuals with Obesity as Compared to the Peripheral Blood

Our previously published findings on the expression of SASP markers in B cells from the peripheral blood of young and elderly individuals have shown that these markers are almost exclusively expressed in memory B cells. We therefore measured the frequencies of naïve and memory B cell subsets in the blood and in the AT of our participants. We used anti-IgD and anti-CD27 antibodies to identify the four major subsets of B cells: naïve $(\operatorname{IgD}+\mathrm{CD} 27-)$, IgM memory $(\operatorname{IgD}+\mathrm{CD} 27+)$, switched memory (IgD-CD27+), and double negative memory (DN, IgD-CD27-) B cells. Results in Figure 2A show gating strategies to evaluate frequencies of naïve and memory B cells in the blood and in the AT of individuals with obesity. Results in Figure 2B show higher frequencies of memory B cells [that include the subsets of IgM memory, switched memory and DN B cells], and reduced frequencies of naïve B cells, in the AT as compared to the blood of individuals with obesity. Naïve B cell frequencies in the blood of obese versus lean individuals were decreased, although not significantly (42 \pm 4 versus $53 \pm 4, p=0.05$ ), whereas memory B cells were significantly higher ( $58 \pm 2$ versus $47 \pm 3(p=0.04)$. These results altogether suggest that the increased expression of SASP markers in B cells from the blood of obese versus lean individuals, 
as well as in B cells from AT versus blood B cells, is likely dependent on the increased frequency of memory B cell subsets.
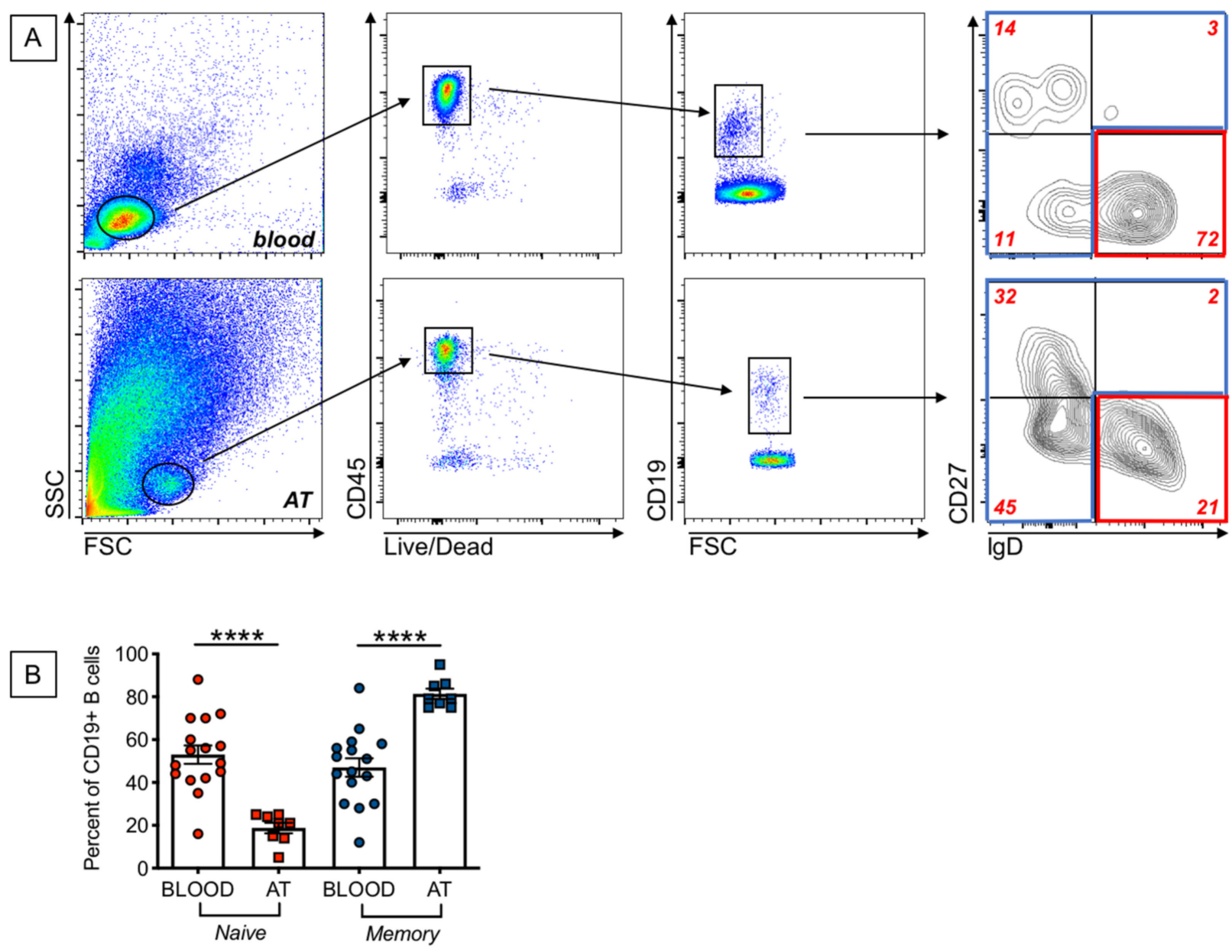

Figure 2. Increased frequencies of memory B cells, and decreased frequencies of naïve B cells, in the AT, as compared to the peripheral blood, of individuals with obesity. (A). Gating strategies and a representative dot plot from PBMC (top) and SVF (bottom) to show naïve and memory B cell subsets. (B). Frequencies of naïve and the memory B cell subsets that include IgM memory, switched memory and DN memory B cells. Mean comparisons between groups were performed by Student's $t$ test (two-tailed). ${ }^{* * * *} p<0.0001$.

\subsection{B Cells from the AT of Individuals with Obesity Are Highly Metabolic as Compared to Those from the Peripheral Blood}

Senescent cells have been shown to be metabolically active, a condition necessary to support the SASP. We evaluated the metabolic profile of B cells from the blood and the AT of individuals with obesity, which depends on uptake of nutrients. We, herefore, measured the uptake of glucose and lipids, by flow cytometry, using the glucose analog (2-(N-(7-Nitrobenz-2-oxa-1,3-diazol-4-yl)Amino)-2-Deoxyglucose) (2-NBDG) and the Deep Red Neutral Lipid compound LipidTOX, respectively. Results show increased glucose uptake (Figure 3A) and increased lipid uptake (Figure 3B) in B cells from the AT as compared to $\mathrm{B}$ cells from the blood. When we compared glucose uptake in B cells from the blood of lean and obese individuals, we found significantly increased 2-NBDG staining in $B$ cells from obese versus lean individuals (1073 \pm 46 , versus $741 \pm 40, p=0.0003$ ). Similar results were obtained when we measured lipid uptake by LipidTOX staining $(8071 \pm 353$ versus $5804 \pm 615, p=0.004)$. Results in Figure $3 C$ also show increased RNA expression of enzymes involved in metabolic pathways, such as HK2, hexokinase 2, a key glycolytic enzyme that phosphorylates glucose; LDHA, lactate dehydrogenase, that converts pyruvate into lactate and represents a measure of anaerobic glycolysis; PDHX, a component of the 
pyruvate dehydrogenase complex that converts pyruvate into acetyl-CoA and represents a measure of oxidative phosphorylation and mitochondrial function; ACACB, acethylCoA carboxylase, a regulator of fatty acid synthesis. Values in B cells from the blood of obese versus lean individuals were: $\mathrm{HK} 2,0.27 \pm 0.01$ versus $0.18 \pm 0.01(p=0.0001)$; LDHA, $0.18 \pm 0.01$ versus $0.10 \pm 0.001(p=0.0001)$; PDHX, $0.18 \pm 0.012(p=0.0001)$ versus $0.12 \pm 0.01 ; \mathrm{ACACB}, 0.29 \pm 0.01$ versus $0.25 \pm 0.01(p=0.005)$. These results altogether suggest that obesity induces $B$ cells characterized by a higher metabolic profile which is needed to support their increased IA and function, including the production of pro-inflammatory mediators that support local and systemic inflammation. The higher metabolic phenotype of B cells from the AT is also associated with increased secretion of $\operatorname{IgG}$ antibodies with autoimmune specificity, as we have previously shown $[29,30]$.
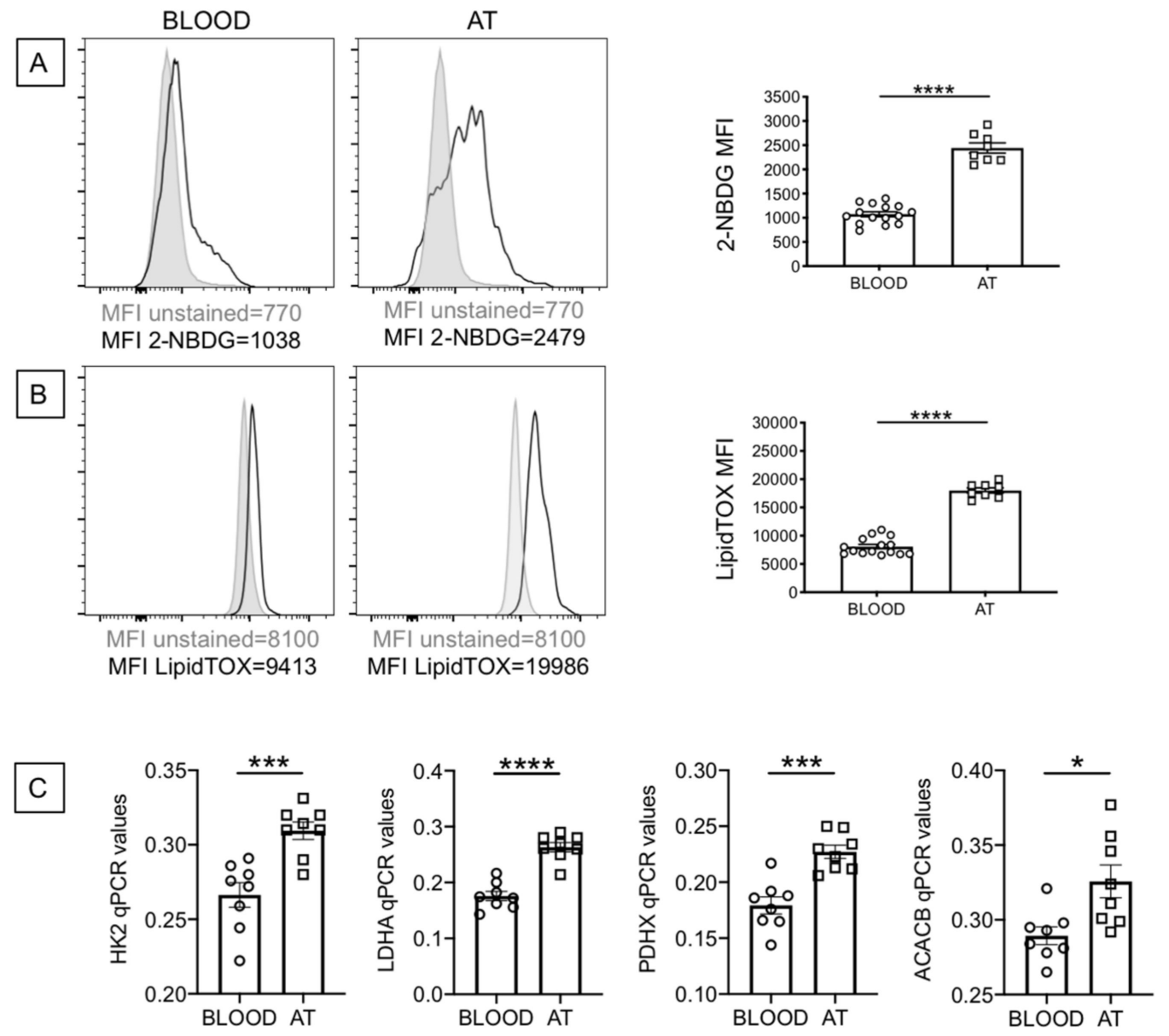

Figure 3. B cells from the AT of individuals with obesity are highly metabolic as compared to those from the peripheral blood. (A) Glucose uptake was measured by flow cytometry and the glucose fluorescent analog 2-NBDG.Results show the MFI (mean fluorescence intensity) profile of one representative blood donor and one representative AT donor (left 2 panels) and data from all donors (right). (B) Lipid uptake was measured by flow cytometry and the Deep Red Neutral Lipid Stain LipidTOX. Results show the MFI profile of one representative blood donor and one representative AT donor (left 2 panels) and data from all donors (right). (C) The mRNA was extracted and qPCR performed to evaluate expression of HK2, LDHA, PDHX and ACACB. qPCR values are measures of RNA expression of target genes, relative to the housekeeping gene GAPDH, calculated as $2^{-\Delta \mathrm{Cts}}$. Mean comparisons between groups were performed by Student's $t$ test (two-tailed). ${ }^{*} p<0.05$, $* * * p<0.001, * * * * x<0.0001$. 


\section{Discussion and Conclusions}

The comprehensive investigation of the contribution of senescent B cells to AT dysfunction is warranted. Results in this manuscript have identified and characterized B cells with a SASP phenotype in the AT of patients undergoing weight reduction surgeries, as compared to those from the peripheral blood of individuals with obesity (age- and gendermatched). Results show increased expression of several SASP markers (pro-inflammatory cytokines, chemokines and miRs, as well as markers of cell cycle arrest) in B cells from the blood of obese versus lean individuals, confirming and extending our previously published findings [18]. Moreover, these SASP markers are expressed at higher levels in B cells from the AT as compared to those from the peripheral blood, providing the first evidence of the presence of senescent B cells in the human AT. Our results also show that AT-derived B cells are hyper-metabolic as compared to blood-derived B cells. This metabolic reprogramming is necessary to support increased immune activation and secretion of SASP factors that fuel local and systemic inflammation, as it has already been shown [31]. Cells with a senescent phenotype are known to have an altered metabolism associated with increased oxidative stress, impairment of specific metabolic pathways and accumulation of oxidized proteins [32]. Metabolic reprogramming is therefore required for these cells to cope with the energetic demands of the senescent program that include the increased secretion of SASP factors, increased oxidative stress and increased endoplasmic reticulum stress.

Cellular senescence indicates the cell cycle arrest elicited in response to a variety of stressors. Despite their inability to proliferate, senescent cells are transcriptionally and metabolically active and secrete multiple SASP factors [3]. In this paper we measured TNF and IL-6 RNA expression because these pro-inflammatory cytokines are secreted at high levels by both the adipocytes and the immune cells in the obese AT, and regulate lipolysis and local inflammation, as we have previously demonstrated [30]. We measured RNA expression of the chemokine IL- 8 because is secreted in large amounts by the obese AT [30] and is responsible for the recruitment of both naïve and memory B cell subsets [33]. For the miRs, we selected miR-155, miR-16 and miR-181a because they regulate AT inflammation and adipocyte differentiation [34-36]. We also measured RNA expression of the canonical marker of senescence $\mathrm{p} 16^{\mathrm{INK} 4}$, associated with cell cycle arrest in senescent B cells, as we have previously shown [37], as well as of the other cell cycle regulators p21 CIP1/WAF1 and $\mathrm{p} 53$.

The AT of obese human beings is characterized by increased oxidative stress, measured by accumulation of intracellular reactive oxygen species (ROS) [38], known to accelerate senescence of AT-derived cells, as indicated by the finding that intracellular ROS activates p38 mitogen-activated protein kinases (MAPKs), which in turn induce p53/p21 CIP1/WAF1 dependent senescence [39]. Telomere shortening, another mechanism of cell senescence, has also been shown in the AT [40]. Although the majority of studies have evaluated the senescent phenotype of non-immune cells (pre-adipocytes and adipocytes) in the obese AT, we show here that AT-derived B cells also have a senescent phenotype, confirming recent findings showing high inflammatory mouse AT-B cells, whose expansion is dependent on the activation of the NLRP3 inflammasome [41].

$\mathrm{B}$ cells are among the first immune cell types that infiltrate the obese AT in both mice [41-43] and humans [30], where they secrete pro-inflammatory mediators, regulate inflammatory $\mathrm{T}$ cells and macrophages, and secrete pathogenic IgG antibodies with autoimmune specificities [29,43]. Therefore, depletion of systemic [43] or AT-derived [41] B cells, through intra-peritoneal or intra-AT injection with an anti-CD20 depleting monoclonal antibody, has shown to significantly improve metabolic and immunological function of the tissue in mice. In both cases, mice injected with the anti-CD20 antibody showed a reduction in pathogenic $B$ cell numbers and function, as well as increased glucose and insulin tolerance. Moreover, consistent with a role for B cells in regulating the AT microenvironment through the modulation of inflammatory $\mathrm{T}$ cells and macrophages, significantly decreased secretion of the key pro-inflammatory mediators IFN- $\gamma$ and TNF- $\alpha$ was observed in the AT of anti-CD20-treated mice as compared to isotype-treated controls. These 
results altogether demonstrate the importance of $B$ cell depletion therapies and support the need to identify additional ways to block hyper-inflammatory and hyper-metabolic $B$ cells that feed-forward local and systemic inflammation, and alleviate obesity-associated metabolic and immunological dysfunction. This represents an important step towards the improvement of the biological quality of life in the obese population.

In conclusion, our results have identified and characterized senescent B cells in the AT of individuals undergoing weight reduction surgery, as compared to those in the peripheral blood of individuals with obesity, thus providing the first evidence of the presence of senescent B cells in the human obese AT. Our results have also identified dysregulated metabolic pathways associated with the SASP. These pathways/markers may be targeted to improve immune function not only in the obese population but also in the general population. Importantly, these interventions will have a significant impact in the management of obesity and related comorbidities, reducing the risk of chronic disease and adverse health outcomes in this vulnerable population.

\section{Materials and Methods}

\subsection{Subjects}

Experiments were conducted using the obese subcutaneous AT obtained from adult female donors undergoing breast reduction surgery $[n=8$, age $=40-55$ years, body-mass index (BMI) $\geq 35 \mathrm{~kg} / \mathrm{m}^{2}$ ] at the Division of Plastic and Reconstructive Surgery at the University of Miami Hospital. As controls, we used peripheral blood from age-, genderand BMI-matched individuals with obesity $(n=18)$. Study participants provided written informed consent. The study was reviewed and approved by the Institutional Review Board (IRB, protocols \#20070481 and \#20160542), which reviews all human research conducted under the auspices of the University of Miami. We enrolled participants without cancer, Congestive Heart Failure, Cardiovascular Disease, Chronic Renal Failure, renal or hepatic diseases, autoimmune diseases, infectious disease as well as individuals without recent ( $<3$ months) trauma or surgery, pregnancy, or documented current substance abuse.

\subsection{Isolation and Processing of the AT}

The AT isolated from surgery patients was harvested, weighed and washed with $1 \times$ Hanks' Balanced Salt Solution (HBSS). It was then resuspended in Dulbecco's modified Eagle's medium (DMEM), minced into small pieces, passed through a $70 \mu \mathrm{m}$ filter and digested with collagenase type I (SIGMA C-9263) for $1 \mathrm{~h}$ in a $37^{\circ} \mathrm{C}$ water bath. Digested cells were passed through a $300 \mu \mathrm{m}$ filter, centrifuged at $300 \times g$ in order to separate the floating adipocytes from the stromal vascular fraction (SVF) containing the immune cells. The cells floating on the top were transferred to a new tube as adipocytes. The cell pellet (SVF) on the bottom was resuspended in ACK for $3 \mathrm{~min}$ at RT (room temperature) to lyse the Red Blood Cells. The SVF was washed 3 times with DMEM. Nucleated cells were counted in a hemocytometer.

\subsection{Flow Cytometry}

Peripheral Blood Mononuclear Cells (PBMC) and SVF from obese donors (10 cells) were stained for $20 \mathrm{~min}$ at room temperature (RT) with the following antibodies: Live/Dead detection kit (InVitrogen1878898), anti-CD45 (Biolegend 368540) and anti-CD19 (BD 555415). To evaluate naïve and memory B cells, PBMC and SVF were stained with anti-CD19, antiCD27 (BD 555441) and anti-IgD (BD 555778) to measure naive (IgD + CD27-) and all memory B cells that include the subsets of IgM memory (IgD + CD27+), switched memory (IgD-CD27+), and double negative memory (DN, IgD-CD27-) B cells. After staining, red blood cells were lyzed using the RBC Lysing Solution (BD 555899), according to the manufacturer's instructions. Up to $10^{5}$ events in the lymphocyte gate were acquired on an LSR-Fortessa (BD) and analyzed using FlowJo 10.5.3 software. Single color controls were included in every experiment for compensation. Isotype controls were also used in every experiment to set up the gates. 


\subsection{B Cell Sorting}

PBMC from individuals with obesity were collected using Vacutainer CPT tubes (BD $362761)$ and cryopreserved. PBMC $\left(1 \times 10^{6} / \mathrm{mL}\right)$ were thawed and cultured in complete medium (c-RPMI, RPMI 1640, supplemented with 10\% FCS, $10 \mu \mathrm{g} / \mathrm{mL}$ Pen-Strep, $1 \mathrm{mM}$ Sodium Pyruvate, and $2 \times 10^{-5} \mathrm{M}$ 2-ME and $2 \mathrm{mM}$ L-glutamine). B cells were isolated from PBMC using magnetic CD19 Microbeads (Miltenyi), following manufacturer's instructions.

For B cell isolation from the AT, the SVF was stained with anti-CD45 and anti-CD19 antibodies. CD19+ B cells were sorted in a Sony SH800 cell sorter. Cell preparations were typically $>98 \%$ pure.

\subsection{RNA Extraction and Quantitative (q)PCR}

To evaluate the expression of SASP markers, B cells were resuspended in TRIzol (Ambion) $\left(10^{6}\right.$ cells $\left./ 100 \mu \mathrm{L}\right)$, then RNA extracted for quantitative (q)PCR. Total RNA was isolated according to the manufacturer's protocol, eluted into $10 \mu \mathrm{L}$ of pre-heated elution buffer and stored at $-80^{\circ} \mathrm{C}$ until use. Reverse transcriptase (RT) reactions were performed in a Mastercycler Eppendorf Thermocycler to obtain cDNA. Briefly, $10 \mu \mathrm{L}$ of mRNA or $2 \mu \mathrm{L}$ of RNA at the concentration of $0.5 \mu \mathrm{g} / \mu \mathrm{L}$ were used as template for cDNA synthesis in the RT reaction. For miRs quantification, RNA was reverse transcribed in the presence of specific primers (provided together with the qPCR primers, see below). In both cases, conditions were: $40 \mathrm{~min}$ at $42{ }^{\circ} \mathrm{C}$ and $5 \mathrm{~min}$ at $65^{\circ} \mathrm{C}$.

To evaluate RNA expression of enzymes involved in metabolic pathways, the mRNA was extracted from B cells, using the $\mu$ MACS mRNA isolation kit (Miltenyi), according to the manufacturer's protocol, eluted into $75 \mu \mathrm{L}$ of pre-heated elution buffer, and stored at $-80{ }^{\circ} \mathrm{C}$ until use. The mRNA was reverse transcribed with the same conditions as above.

qPCR reactions were conducted in MicroAmp 96-well plates and run in the ABI 7300 machine. Calculations were made with ABI software. Briefly, we determined the cycle number at which transcripts reached a significant threshold $(\mathrm{Ct})$ for each target gene, and for GAPDH or U6 as controls. The difference in Cts between the housekeeping genes (GAPDH or U6) and the target genes was calculated as $\triangle \mathrm{Ct}$. Then the relative amount of the target gene was expressed as $2^{-\Delta C t}$ and indicated as $\mathrm{qPCR}$ values. Reagents and Taqman primers, all from Life Technologies, were the following: GAPDH, Hs99999905_m1; TNF, Hs01113624_g1; IL-6, Hs00985639_m1; IL-8, Hs00174103_m1; p16 ${ }^{\text {INK4 }}$ (CDKN2A), Hs00923894_m1; p21 CIP1/WAF1, Hs00355782_m1; p53, Hs01034249_m1. HK2, Hs00606086_m1; LDHA, Hs01378790_g1; PDHX, Hs00185790_m1; ACACB, Hs01565914_m1; U6, 001973; miR-155, 002623; miR-16, 000391; miR-181a, 000480.

\subsection{Glucose and Lipid Uptake Measurements}

PBMCs $\left(10^{6} / \mathrm{mL}\right)$ and SVF were stained with the fluorescent glucose analog (2(N-(7-Nitrobenz-2-oxa-1,3-diazol-4-yl)Amino)-2-Deoxyglucose) (2-NBDG, Thermo Fisher N13195), or with the Deep Red Neutral Lipid Stain LipidTOX (Thermo Fisher H34476), for $30 \mathrm{~min}$ at RT, at the final concentrations recommended by the manufacturer. Cells were then washed and stained for $20 \mathrm{~min}$ at room temperature with anti-CD45, anti-CD19, as well as with the Live/Dead detection kit. Cells were washed and later acquired in a BD LSR Fortessa Flow cytometry instrument, using the FITC channel to detect the signal from the fluorescent glucose uptake tracker and the APC channel to detect the signal from the LipidTOX. Fluorescence data were analyzed using FlowJo 10.0.6 software.

\subsection{Statistical Analyses}

Mean comparisons were performed by unpaired Student's $t$ tests (two-tailed), using GraphPad Prism version 8 software, which was used to construct all graphs.

Author Contributions: D.F.: conceptualization, data curation, formal analysis, investigation, methodology, funding acquisition, writing-original draft, writing-review and editing. M.R.: data curation, formal analysis, investigation, methodology, writing-review and editing. A.D.: data curation, formal 
analysis, investigation, methodology, writing — review and editing. D.G.: investigation, methodology, writing-review and editing. S.T.: methodology, writing-review and editing. B.B.B.: conceptualization, funding acquisition, writing-review and editing. All authors have read and agreed to the published version of the manuscript.

Funding: This study was supported by NIH awards AG32576, AG059719, AG023717.

Institutional Review Board Statement: The study was conducted according to the guidelines of the Declaration of Helsinki, reviewed and approved by the Institutional Review Board (IRB, protocols \#20070481 and \#20160542), which reviews all human research conducted under the auspices of the University of Miami.

Informed Consent Statement: Informed consent was obtained from all subjects involved in the study.

Data Availability Statement: The data presented in this study are available upon request to the corresponding author.

Acknowledgments: We thank the volunteers who have participated in this study, the personnel of the University of Miami Hospital for the recruitment of the participants and the Sylvester Comprehensive Cancer Center (SCCC) Flow Cytometry Core Resource. We also thank all members of our laboratory for critical discussions.

Conflicts of Interest: The authors declare no conflict of interest. The funders had no role in the design of the study; in the collection, analyses, or interpretation of data; in the writing of the manuscript, or in the decision to publish the results.

\section{References}

1. Childs, B.G.; Durik, M.; Baker, D.J.; Van Deursen, J.M. Cellular Senescence in Aging and Age-Related Disease: From Mechanisms to Therapy. Nat. Med. 2015, 21, 1424-1435. [CrossRef] [PubMed]

2. Van Deursen, J.M. The Role of Senescent Cells in Ageing. Nat. Cell Biol. 2014, 509, 439-446. [CrossRef]

3. Campisi, J. Cellular Senescence: Putting the Paradoxes in Perspective. Curr. Opin. Genet. Dev. 2011, 21, 107-112. [CrossRef] [PubMed]

4. Sikora, E.; Arendt, T.; Bennett, M.; Narita, M. Impact of Cellular Senescence Signature on Ageing Research. Ageing Res. Rev. 2011, 10, 146-152. [CrossRef] [PubMed]

5. Campisi, J. Cellular Senescence and Apoptosis: How Cellular Responses Might Influence Aging Phenotypes. Exp. Gerontol. 2003, 38, 5-11. [CrossRef]

6. Passos, J.F.; Nelson, G.; Wang, C.; Richter, T.; Simillion, C.; Proctor, C.J.; Miwa, S.; Olijslagers, S.; Hallinan, J.; Wipat, A.; et al. Feedback between p21 and Reactive Oxygen Production Is Necessary for Cell Senescence. Mol. Syst. Biol. 2010, 6, 347. [CrossRef] [PubMed]

7. Frasca, D.; Blomberg, B.B. Adipose Tissue, Immune Aging, and Cellular Senescence. Semin. Immunopathol. 2020, 42, 573-587. [CrossRef]

8. Liu, Z.; Wu, K.K.; Jiang, X.; Xu, A.; Cheng, K.K. The Role of Adipose Tissue Senescence in Obesity- and Ageing-Related Metabolic Disorders. Clin. Sci. 2020, 134, 315-330. [CrossRef]

9. Tchkonia, T.; Morbeck, D.E.; Von Zglinicki, T.; Van Deursen, J.; Lustgarten, J.; Scrable, H.; Khosla, S.; Jensen, M.D.; Kirkland, J.L. Fat Tissue, Aging, and Cellular Senescence. Aging Cell 2010, 9, 667-684. [CrossRef]

10. Franceschi, C.; Bonafè, M.; Valensin, S.; Olivieri, F.; De Luca, M.; Ottaviani, E.; De Benedictis, G. Inflammaging: An Evolutionary Perspective on Immunosenescence. Ann. N. Y. Acad. Sci. 2000, 908, 244-254. [CrossRef]

11. Hotamisligil, G.S. Inflammation and Metabolic Disorders. Nat. Cell Biol. 2006, 444, 860-867. [CrossRef]

12. Johnson, A.M.; Olefsky, J.M. The Origins and Drivers of Insulin Resistance. Cell 2013, 152, 673-684. [CrossRef]

13. Shoelson, S.E.; Lee, J.; Goldfine, A.B. Inflammation and Insulin Resistance. J. Clin. Investig. 2006, $116,1793-1801$. [CrossRef] [PubMed]

14. Apovian, C.M.; Gokce, N. Obesity and Cardiovascular Disease. Circulation 2012, 125, 1178-1182. [CrossRef]

15. Renehan, A.G.; Tyson, M.; Egger, M.; Heller, R.F.; Zwahlen, M. Body-Mass Index and Incidence of Cancer: A Systematic Review and Meta-Analysis of Prospective Observational Studies. Lancet 2008, 371, 569-578. [CrossRef]

16. Casas, R.; Sacanella, E.; Estruch, R. The Immune Protective Effect of the Mediterranean Diet against Chronic Low-Grade Inflammatory Diseases. Endocrine Metab. Immune Disord. Drug Targets 2014, 14, 245-254. [CrossRef] [PubMed]

17. Hass, D.J.; Brensinger, C.M.; Lewis, J.D.; Lichtenstein, G.R. The Impact of Increased Body Mass Index on the Clinical Course of Crohn's Disease. Clin. Gastroenterol. Hepatol. 2006, 4, 482-488. [CrossRef]

18. Frasca, D.; Ferracci, F.; Diaz, A.; Romero, M.; Lechner, S.; Blomberg, B.B. Obesity Decreases B Cell Responses in Young and Elderly Individuals. Obesity 2016, 24, 615-625. [CrossRef]

19. Ovsyannikova, I.G.; White, S.J.; Larrabee, B.R.; Grill, D.E.; Jacobson, R.M.; Poland, G.A. Leptin and Leptin-Related Gene Polymorphisms, Obesity, and Influenza A/H1N1 Vaccine-Induced Immune Responses in Older Individuals. Vaccine 2014, 32, 881-887. [CrossRef] 
20. Sheridan, P.A.; Paich, H.A.; Handy, J.; Karlsson, E.A.; Hudgens, M.G.; Sammon, A.B.; Holland, L.A.; Weir, S.; Noah, T.L.; Beck, M.A. Obesity Is Associated with Impaired Immune Response to Influenza Vaccination in Humans. Int. J. Obes. 2011, 36, 1072-1077. [CrossRef]

21. George, M.; Baker, J.F. The Obesity Epidemic and Consequences for Rheumatoid Arthritis Care. Curr. Rheumatol. Rep. 2016, 18, 1-9. [CrossRef]

22. Ottaviani, S.; Allanore, Y.; Tubach, F.; Forien, M.; Gardette, A.; Pasquet, B.; Palazzo, E.; Meunier, M.; Hayem, G.; Job-Deslandre, C.; et al. Body Mass Index Influences the Response to Infliximab in Ankylosing Spondylitis. Arthritis Res. Ther. 2012, 14, R115. [CrossRef]

23. Sandberg, M.E.C.; Bengtsson, C.; Källberg, H.; Wesley, A.; Klareskog, L.; Alfredsson, L.; Saevarsdottir, S. Overweight Decreases the Chance of Achieving Good Response and Low Disease Activity in Early Rheumatoid Arthritis. Ann. Rheum. Dis. 2014, 73, 2029-2033. [CrossRef]

24. Muramatsu, M.; Kinoshita, K.; Fagarasan, S.; Yamada, S.; Shinkai, Y.; Honjo, T. Class Switch Recombination and Hypermutation Require Activation-Induced Cytidine Deaminase (AID), a Potential RNA Editing Enzyme. Cell 2000, 102, 553-563. [CrossRef]

25. Frasca, D.; Diaz, A.; Romero, M.; Landin, A.M.; Phillips, M.; Lechner, S.C.; Ryan, J.G.; Blomberg, B.B. Intrinsic Defects in B Cell Response to Seasonal Influenza Vaccination in Elderly Humans. Vaccine 2010, 28, 8077-8084. [CrossRef]

26. Frasca, D.; Diaz, A.; Romero, M.; Phillips, M.; Mendez, N.V.; Landin, A.M.; Blomberg, B.B. Unique Biomarkers for B-Cell Function Predict the Serum Response to Pandemic H1N1 Influenza Vaccine. Int. Immunol. 2012, 24, 175-182. [CrossRef]

27. Khurana, S.; Frasca, D.; Blomberg, B.; Golding, H. AID Activity in B Cells Strongly Correlates with Polyclonal Antibody Affinity Maturation In-Vivo Following Pandemic 2009-H1N1 Vaccination in Humans. PLOS Pathog. 2012, 8, e1002920. [CrossRef] [PubMed]

28. Lee, Y.-H.; Kim, S.R.; Han, D.H.; Yu, H.T.; Han, Y.D.; Kim, J.H.; Kim, S.H.; Lee, C.J.; Min, B.-H.; Kim, D.-H.; et al. Senescent T Cells Predict the Development of Hyperglycemia in Humans. Diabetes 2019, 68, 156-162. [CrossRef]

29. Frasca, D.; Diaz, A.; Romero, M.; Garcia, D.; Jayram, D.; Thaller, S.; Piqueras, M.D.C.; Bhattacharya, S.; Blomberg, B.B. Identification and Characterization of Adipose Tissue-Derived Human Antibodies With "Anti-Self" Specificity. Front. Immunol. 2020, 11, 392. [CrossRef]

30. Frasca, D.; Diaz, A.; Romero, M.; Thaller, S.; Blomberg, B.B. Secretion of Autoimmune Antibodies in the Human Subcutaneous Adipose Tissue. PLoS ONE 2018, 13, e0197472. [CrossRef] [PubMed]

31. Wiley, C.D.; Campisi, J. From Ancient Pathways to Aging Cells-Connecting Metabolism and Cellular Senescence. Cell Metab. 2016, 23, 1013-1021. [CrossRef]

32. Hamon, M.; Ahmed, E.K.; Baraibar, M.A.; Friguet, B. Proteome Oxidative Modifications and Impairment of Specific Metabolic Pathways During Cellular Senescence and Aging. Proteomics 2019, 20, e1800421. [CrossRef] [PubMed]

33. Henneken, M.; Dörner, T.; Burmester, G.-R.; Berek, C. Differential Expression of Chemokine Receptors on Peripheral Blood B Cells from Patients with Rheumatoid Arthritis and Systemic Lupus Erythematosus. Arthritis Res. Ther. 2005, 7, R1001-R1013. [CrossRef] [PubMed]

34. Arner, P.; Kulyté, A. MicroRNA Regulatory Networks in Human Adipose Tissue and Obesity. Nat. Rev. Endocrinol. 2015, 11, 276-288. [CrossRef] [PubMed]

35. Deiuliis, A.J. MicroRNAs as Regulators of Metabolic Disease: Pathophysiologic Significance and Emerging Role as Biomarkers and Therapeutics. Int. J. Obes. 2016, 40, 88-101. [CrossRef]

36. Iacomino, G.; Siani, A. Role of MicroRNAs in Obesity and Obesity-Related Diseases. Genes Nutr. 2017, 12, 1-16. [CrossRef] [PubMed]

37. Frasca, D.; Diaz, A.; Romero, M.; Blomberg, B.B. Human Peripheral Late/Exhausted Memory B Cells Express a SenescentAssociated Secretory Phenotype and Preferentially Utilize Metabolic Signaling Pathways. Exp. Gerontol. 2017, 87, 113-120. [CrossRef]

38. Furukawa, S.; Fujita, T.; Shimabukuro, M.; Iwaki, M.; Yamada, Y.; Nakajima, Y.; Nakayama, O.; Makishima, M.; Matsuda, M.; Shimomura, I. Increased Oxidative Stress in Obesity and Its Impact on Metabolic Syndrome. J. Clin. Investig. 2004, 114, 1752-1761. [CrossRef]

39. Passos, J.F.; Simillion, C.; Hallinan, J.; Wipat, A.; Von Zglinicki, T. Cellular Senescence: Unravelling Complexity. AGE 2009, 31, 353-363. [CrossRef]

40. Valdes, A.M.; Andrew, T.; Gardner, J.P.; Kimura, M.; Oelsner, E.; Cherkas, L.F.; Aviv, A.; Spector, T.D. Obesity, Cigarette Smoking, and Telomere Length in Women. Lancet 2005, 366, 662-664. [CrossRef]

41. Camell, C.D.; Günther, P.; Lee, A.; Goldberg, E.L.; Spadaro, O.; Youm, Y.-H.; Bartke, A.; Hubbard, G.B.; Ikeno, Y.; Ruddle, N.H.; et al. Aging Induces an Nlrp3 Inflammasome-Dependent Expansion of Adipose B Cells That Impairs Metabolic Homeostasis. Cell Metab. 2019, 30, 1024-1039.e6. [CrossRef] [PubMed]

42. Frasca, D.; Diaz, A.; Romero, M.; Vazquez, T.; Blomberg, B.B. Obesity Induces Pro-inflammatory B Cells and Impairs B Cell Function in Old Mice. Mech. Ageing Dev. 2017, 162, 91-99. [CrossRef] [PubMed]

43. Winer, D.A.; Winer, S.; Shen, L.; Wadia, P.P.; Yantha, J.; Paltser, G.; Tsui, H.; Wu, P.; Davidson, M.G.; Alonso, M.N.; et al. B Cells Promote Insulin Resistance through Modulation of T Cells and Production of Pathogenic Igg Antibodies. Nat. Med. 2011, 17, 610-617. [CrossRef] [PubMed] 